\title{
A survey on the oscillation of differential equations with several deviating arguments
}

\author{
loannis P Stavroulakis*
}

Dedicated to Professor Ivan T Kiguradze, an excellent scientist and very good friend and colleague

"Correspondence: ipstav@cc.uoi.gr Department of Mathematics, University of loannina, loannina, 451 10, Greece

\section{Abstract}

Consider the first-order linear differential equation with several retarded arguments

$$
x^{\prime}(t)+\sum_{i=1}^{m} p_{i}(t) x\left(\tau_{i}(t)\right)=0, \quad t \geq t_{0}
$$

where the functions $p_{i}, \tau_{i} \in C\left(\left[t_{0}, \infty\right), \mathbb{R}^{+}\right)$for every $i=1,2, \ldots, m, \tau_{i}(t) \leq t$ for $t \geq t_{0}$ and $\lim _{t \rightarrow \infty} \tau_{i}(t)=\infty$. A survey on the oscillation of all solutions to this equation is presented in the case of several non-monotone arguments and especially when well-known oscillation conditions are not satisfied. Examples illustrating the results are given.

MSC: Primary 34K11; secondary 34K06

Keywords: oscillation; retarded; differential equations; non-monotone arguments

\section{Introduction}

Consider the differential equation with several non-monotone retarded arguments

$$
x^{\prime}(t)+\sum_{i=1}^{m} p_{i}(t) x\left(\tau_{i}(t)\right)=0, \quad t \geq t_{0},
$$

where the functions $p_{i}, \tau_{i} \in C\left(\left[t_{0}, \infty\right), \mathbb{R}^{+}\right)$for every $i=1,2, \ldots, m$ (here $\left.\mathbb{R}^{+}=[0, \infty)\right), \tau_{i}(t) \leq$ $t$ for $t \geq t_{0}$ and $\lim _{t \rightarrow \infty} \tau_{i}(t)=\infty$.

Let $T_{0} \in\left[t_{0},+\infty\right), \tau(t)=\min \left\{\tau_{i}(t): i=1, \ldots, m\right\}$ and $\tau_{(-1)}(t)=\inf \{\tau(s): s \geq t\}$. By a solution of equation (1.1), we understand a function $u \in C\left(\left[t_{0},+\infty\right) ; R\right)$ continuously differentiable on $\left[\tau_{(-1)}\left(T_{0}\right),+\infty\right)$ that satisfies $(1.1)$ for $t \geq \tau_{(-1)}\left(T_{0}\right)$. Such a solution is called oscillatory if it has arbitrarily large zeros, and otherwise it is called non-oscillatory.

In the special case where $m=1$, equation (1.1) reduces to the equation

$$
x^{\prime}(t)+p(t) x(\tau(t))=0, \quad t \geq t_{0},
$$

where the functions $p, \tau \in C\left(\left[t_{0}, \infty\right), \mathbb{R}^{+}\right), \tau(t) \leq t$ for $t \geq t_{0}$ and $\lim _{t \rightarrow \infty} \tau(t)=\infty$.

For the general theory of these equations, the reader is referred to [1-4].

C2014 Stavroulakis; licensee Springer. This is an Open Access article distributed under the terms of the Creative Commons Attribution License (http://creativecommons.org/licenses/by/2.0), which permits unrestricted use, distribution, and reproduction in any medium, provided the original work is properly cited. 
In this paper we present a survey on the oscillation of all solutions to these equations in the case of several non-monotone arguments and especially when the well-known oscillation conditions

$$
\limsup _{t \rightarrow \infty} \int_{\tau(t)}^{t} p(s) d s>1 \text { and } \quad \liminf _{t \rightarrow \infty} \int_{\tau(t)}^{t} p(s) d s>\frac{1}{e}
$$

for equation (1.2) are not satisfied.

\section{Oscillation criteria for equation (1.2)}

In this section we study the delay equation

$$
x^{\prime}(t)+p(t) x(\tau(t))=0, \quad t \geq t_{0},
$$

where the functions $p, \tau \in C\left(\left[t_{0}, \infty\right), \mathbb{R}^{+}\right), \tau(t)<t$ for $t \geq t_{0}$ and $\lim _{t \rightarrow \infty} \tau(t)=\infty$.

The problem of establishing sufficient conditions for the oscillation of all solutions to the differential equation (1.2) has been the subject of many investigations. See, for example, $[1,5-26]$ and the references cited therein.

The first systematic study for the oscillation of all solutions to equation (1.2) was made by Myshkis. In 1950 [20] he proved that every solution of equation (1.2) oscillates if

$$
\limsup _{t \rightarrow \infty}[t-\tau(t)]<\infty \quad \text { and } \quad \liminf _{t \rightarrow \infty}[t-\tau(t)] \liminf _{t \rightarrow \infty} p(t)>\frac{1}{e}
$$

In 1972, Ladas et al. [27] proved that the same conclusion holds if in addition $\tau$ is a non-decreasing function and

$\left(C_{1}\right) \quad A:=\limsup _{t \rightarrow \infty} \int_{\tau(t)}^{t} p(s) d s>1$.

In 1979, Ladas [17] established integral conditions for the oscillation of equation (1.2) with constant delay, while in 1982, Koplatadze and Chanturija [14] established the following result. If

$\left(C_{2}\right) \mathfrak{a}:=\liminf _{t \rightarrow \infty} \int_{\tau(t)}^{t} p(s) d s>\frac{1}{e}$

then all solutions of equation (1.2) oscillate; if

$\left(N_{1}\right) \limsup _{t \rightarrow \infty} \int_{\tau(t)}^{t} p(s) d s<\frac{1}{e}$

then equation (1.2) has a non-oscillatory solution.

It is obvious that there is a gap between conditions $\left(C_{1}\right)$ and $\left(C_{2}\right)$ when the limit $\lim _{t \rightarrow \infty} \int_{\tau(t)}^{t} p(s) d s$ does not exist. How to fill this gap is an interesting problem which has been recently investigated by several authors.

In 1988, Erbe and Zhang [10] developed new oscillation criteria by employing the upper bound of the ratio $x(\tau(t)) / x(t)$ for possible non-oscillatory solutions $x(t)$ of equation (1.2). Their result says that all the solutions of equation (1.2) are oscillatory if $0<\mathfrak{a} \leq \frac{1}{e}$ and 
$\left(C_{3}\right) A>1-\frac{\mathfrak{a}^{2}}{4}$

Since then several authors tried to obtain better results by improving the upper bound for $x(\tau(t)) / x(t)$.

In 1991, Jian [12] derived the condition

$\left(C_{4}\right) A>1-\frac{\mathfrak{a}^{2}}{2(1-\mathfrak{a})}$,

while in 1992, Yu et al. [25] obtained the condition

(C $\left.C_{5}\right) A>1-\frac{1-\mathfrak{a}-\sqrt{1-2 \mathfrak{a}-\mathfrak{a}^{2}}}{2}$.

In 1990, Elbert and Stavroulakis [8] and in 1991 Kwong [16], using different techniques, improved $\left(C_{3}\right)$, in the case where $0<\mathfrak{a} \leq \frac{1}{e}$, to the conditions

(C) $A>1-\left(1-\frac{1}{\sqrt{\lambda_{1}}}\right)^{2}$

and

(C) $A>\frac{\ln \lambda_{1}+1}{\lambda_{1}}$,

respectively, where $\lambda_{1}$ is the smaller real root of the equation $\lambda=e^{\mathfrak{a} \lambda}$.

In 1998, Philos and Sficas [21] and in 1999, Zhou and Yu [26] and Jaroš and Stavroulakis [11] derived the conditions

(C $\left.C_{8}\right) A>1-\frac{\mathfrak{a}^{2}}{2(1-\mathfrak{a})}-\frac{\mathfrak{a}^{2}}{2} \lambda_{1}$,

(C) $A>1-\frac{1-\mathfrak{a}-\sqrt{1-2 \mathfrak{a}-\mathfrak{a}^{2}}}{2}-\left(1-\frac{1}{\sqrt{\lambda_{1}}}\right)^{2}$,

and

$\left(C_{10}\right) A>\frac{\ln \lambda_{1}+1}{\lambda_{1}}-\frac{1-\mathfrak{a}-\sqrt{1-2 \mathfrak{a}-\mathfrak{a}^{2}}}{2}$,

respectively.

Consider equation (1.2) and assume that $\tau(t)$ is continuously differentiable and that there exists $\theta>0$ such that $p(\tau(t)) \tau^{\prime}(t) \geq \theta p(t)$ eventually for all $t$. Under this additional assumption, in 2000, Kon et al. [13] and in 2003, Sficas and Stavroulakis [22] established the conditions

(C 11$) A>2 \mathfrak{a}+\frac{2}{\lambda_{1}}-1$,

and

$\left(C_{12}\right) A>\frac{\ln \lambda_{1}-1+\sqrt{5-2 \lambda_{1}+2 \mathfrak{a} \lambda_{1}}}{\lambda_{1}}$, 
respectively. In the case where $\mathfrak{a}=\frac{1}{e}$, then $\lambda_{1}=e$, and $\left(C_{12}\right)$ leads to

$$
A>\frac{\sqrt{7-2 e}}{e} \approx 0.459987065
$$

It is to be noted that for small values of $\mathfrak{a}(\mathfrak{a} \rightarrow 0)$, all previous conditions $\left(C_{3}\right)-\left(C_{11}\right)$ reduce to condition $\left(C_{1}\right)$, i.e., $A>1$. However, condition $\left(C_{12}\right)$ leads to

$$
A>\sqrt{3}-1 \approx 0.732
$$

which is an essential improvement. Moreover, $\left(C_{12}\right)$ improves all the above conditions for all values of $\mathfrak{a} \in\left(0, \frac{1}{e}\right]$. Note that the value of the lower bound on $A$ cannot be less than $\frac{1}{e} \approx 0.367879441$. Thus, the aim is to establish a condition which leads to a value as close as possible to $\frac{1}{e}$.

For illustrative purpose, we give the values of the lower bound on $A$ under these conditions when (i) $\mathfrak{a}=1 / 1,000$ and (ii) $\mathfrak{a}=1 / e$.

(i)

$\begin{array}{lll}\left(C_{3}\right): & 0.999999750 & 0.966166179 \\ \left(C_{4}\right): & 0.999999499 & 0.892951367 \\ \left(C_{5}\right): & 0.999999499 & 0.863457014 \\ \left(C_{6}\right): & 0.999999749 & 0.845181878 \\ \left(C_{7}\right): & 0.999999499 & 0.735758882 \\ \left(C_{8}\right): & 0.999998998 & 0.709011646 \\ \left(C_{9}\right): & 0.999999249 & 0.708638892 \\ \left(C_{10}\right): & 0.999998998 & 0.599215896 \\ \left(C_{11}\right): & 0.999999004 & 0.471517764 \\ \left(C_{12}\right): & 0.733050517 & 0.459987065\end{array}$

We see that condition $\left(C_{12}\right)$ essentially improves all the known results in the literature.

Moreover, it should be pointed out that in 1994, Koplatadze and Kvinikadze [15] improved $\left(C_{5}\right)$ as follows: Assume

$$
\sigma(t):=\sup _{s \leq t} \tau(s), \quad t \geq 0
$$

Clearly $\sigma(t)$ is non-decreasing and $\tau(t) \leq \sigma(t)$ for all $t \geq 0$. Define

$$
\psi_{1}(t)=0, \quad \psi_{i}(t)=\exp \left\{\int_{\tau(t)}^{t} p(\xi) \psi_{i-1}(\xi) d \xi\right\}, \quad i=2,3, \ldots, \text { for } t \in \mathbb{R}^{+}
$$

Then the following theorem was established in [15].

Theorem $2.1([15])$ Let $k \in\{1,2, \ldots\}$ exist such that

$$
\limsup _{t \rightarrow \infty} \int_{\sigma(t)}^{t} p(s) \exp \left\{\int_{\sigma(s)}^{\sigma(t)} p(\xi) \psi_{k}(\xi) d \xi\right\} d s>1-c(\mathfrak{a})
$$


where $\sigma, \psi_{k}, \mathfrak{a}$ are defined by (2.2), (2.4), $\left(C_{2}\right)$, respectively, and

$$
c(\mathfrak{a})= \begin{cases}0 & \text { if } \mathfrak{a}>\frac{1}{e}, \\ \frac{1}{2}\left(1-\mathfrak{a}-\sqrt{1-2 \mathfrak{a}-\mathfrak{a}^{2}}\right) & \text { if } 0<\mathfrak{a} \leq \frac{1}{e} .\end{cases}
$$

Then all solutions of equation (1.2) oscillate.

Concerning the constants 1 and $\frac{1}{e}$ which appear in conditions $\left(C_{1}\right),\left(C_{2}\right)$ and $\left(N_{1}\right)$, in 2011, Berezansky and Braverman [6] established the following theorem.

Theorem 2.2 ([6]) For any $\alpha \in(1 / e, 1)$, there exists a non-oscillatory equation

$$
x^{\prime}(t)+p(t) x(t-\tau)=0, \quad \tau>0
$$

with $p(t) \geq 0$ such that

$$
\limsup _{t \rightarrow \infty} \int_{t-\tau}^{t} p(s) d s=\alpha
$$

Also in 2011, Braverman and Karpuz [7] investigated equation (1.2) in the case of a general argument ( $\tau$ is not assumed monotone) and proved the following.

Theorem 2.3 ([7]) There is no constant $K>0$ such that

$$
\limsup _{t \rightarrow \infty} \int_{\tau(t)}^{t} p(s) d s>K
$$

implies oscillation of equation (1.2) for arbitrary (not necessarily non-decreasing) argument $\tau(t) \leq t$.

Remark 2.1 Observe that, because of condition $\left(N_{1}\right)$, the constant $K$ in (2.4) makes sense for $K>1 / e$.

Moreover, in [7] the following result was established.

Theorem 2.4 ([7]) Assume that

$$
B:=\limsup _{t \rightarrow \infty} \int_{\sigma(t)}^{t} p(s) \exp \left\{\int_{\tau(s)}^{\sigma(t)} p(\xi) d \xi\right\} d s>1,
$$

where $\sigma(t)$ is defined by (2.1). Then all solutions of equation (1.2) oscillate.

Observe that condition (2.5) improves $\left(C_{1}\right)$.

Using the upper bound of the ratio $\frac{x(\tau(t))}{x(t)}$ for possible non-oscillatory solutions $x(t)$ of equation (1.2), presented in $[8,11,13,22]$, the above result was recently essentially improved in [23]. 
Theorem 2.5 ([23]) Assume that $0<\mathfrak{a} \leq \frac{1}{e}$ and

$$
B:=\limsup _{t \rightarrow \infty} \int_{\sigma(t)}^{t} p(s) \exp \left\{\int_{\tau(s)}^{\sigma(t)} p(\xi) d \xi\right\} d s>1-\frac{1}{2}\left(1-\mathfrak{a}-\sqrt{1-2 \mathfrak{a}-\mathfrak{a}^{2}}\right)
$$

where $\sigma(t)$ is defined by (2.1). Then all solutions of equation (1.2) oscillate.

Remark 2.2 ([23]) Observe that as $\mathfrak{a} \rightarrow 0$, then condition (2.6) reduces to (2.5). However, the improvement is clear as $\mathfrak{a} \rightarrow \frac{1}{e}$. Actually, when $\mathfrak{a}=\frac{1}{e}$, the value of the lower bound on $B$ is equal to $\approx 0.863457014$. That is, (2.6) essentially improves (2.5).

Remark 2.3 ([23]) Note that, under the additional assumption that $\tau(t)$ is continuously differentiable and that there exists $\theta>0$ such that $p(\tau(t)) \tau^{\prime}(t) \geq \theta p(t)$ eventually for all $t$ (see $[13,22])$ condition $(2.6)$ of Theorem 2.5 reduces to

$$
B>1-\frac{1}{2}\left(1-\mathfrak{a}-\sqrt{(1-\mathfrak{a})^{2}-4 M}\right),
$$

where $M$ is given by

$$
M=\frac{e^{\lambda \theta \mathfrak{a}}-\lambda \theta \mathfrak{a}-1}{(\lambda \theta)^{2}}
$$

and $\lambda$ is the smaller root of the equation $\lambda=e^{\lambda \mathfrak{a}}$. When $\theta=1$, then from [22] it follows that

$$
\frac{1}{2}\left(1-\mathfrak{a}-\sqrt{(1-\mathfrak{a})^{2}-4 M}\right)=1-\mathfrak{a}-\frac{1}{\lambda}
$$

and in the case that $\mathfrak{a}=\frac{1}{e}$, then $\lambda=e$ and $\left(2.6^{\prime}\right)$ leads to

$$
B>1-\left(1-\frac{2}{e}\right)=\frac{2}{e} \approx 0.735758882
$$

That is, condition $\left(2.6^{\prime}\right)$ essentially improves (2.6) but of course under the additional (stronger) assumptions on $\tau(t)$ and $p(t)$.

The following example illustrates the significance of Theorem 2.5.

Example 2.1 ( $c f .[7,23])$ Consider the equation

$$
x^{\prime}(t)+p(t) x(\tau(t))=0, \quad t \geq 0,
$$

where $p(t)=\frac{0.93}{e}$, and

$$
\tau(t):= \begin{cases}t-1, & t \in[3 n, 3 n+1], \\ -3 t+(12 n+3), & t \in[3 n+1,3 n+2], \\ 5 t-(12 n+13), & t \in[3 n+2,3 n+3] .\end{cases}
$$


We see that

$$
\sigma(t)= \begin{cases}t-1, & t \in[3 n, 3 n+1], \\ 3 n, & t \in[3 n+1,3 n+2.6], \\ 5 t-(12 n+13), & t \in[3 n+2.6,3 n+3] .\end{cases}
$$

Observe that

$$
\begin{gathered}
\mathfrak{a}=\liminf _{t \rightarrow \infty} \int_{\tau(t)}^{t} \frac{0.93}{e} d s=\frac{0.93}{e} \approx 0.34212788<\frac{1}{e}, \\
\limsup _{t \rightarrow \infty} \int_{\sigma(t)}^{t} \frac{0.93}{e} d s=2.6 \frac{0.93}{e}=0.889532488<1 .
\end{gathered}
$$

Moreover, for $n \geq 0$, we have

$$
\begin{aligned}
& \int_{\sigma(3 n+3)}^{3 n+3} \frac{0.93}{e} \exp \left\{\int_{\tau(s)}^{\sigma(3 n+3)} \frac{0.93}{e} d \xi\right\} d s \\
& \quad=\int_{3 n+2}^{3 n+3} \frac{0.93}{e} \exp \left\{\int_{5 s-(12 n+13)}^{3 n+2} \frac{0.93}{e} d \xi\right\} d s \\
& =\int_{3 n+2}^{3 n+3} \frac{0.93}{e} \exp \left\{\frac{4.65}{e}[3 n+3-s]\right\} d s \\
& =\frac{1}{5}\left[\exp \left\{\frac{4.65}{e}\right\}-1\right] \approx 0.906499566<1 .
\end{aligned}
$$

That is, conditions $\left(C_{1}\right),\left(C_{2}\right)$ and $(2.5)$ are not satisfied. Observe, however, that for $\mathfrak{a} \approx$ 0.34212788 ,

$$
1-\frac{1}{2}\left(1-\mathfrak{a}-\sqrt{1-2 \mathfrak{a}-\mathfrak{a}^{2}}\right) \approx 0.893938766
$$

and from (2.10) we see that

$$
\limsup _{t \rightarrow \infty} \int_{\sigma(t)}^{t} p(s) \exp \left\{\int_{\tau(s)}^{\sigma(t)} p(\xi) d \xi\right\} d s>0.90>1-\frac{1}{2}\left(1-\mathfrak{a}-\sqrt{1-2 \mathfrak{a}-\mathfrak{a}^{2}}\right) \approx 0.89
$$

that is, the conditions of Theorem 2.5 are satisfied and therefore all the solutions of equation (2.7) with the general argument (2.8) are oscillatory.

\section{Oscillation criteria for equation (1.1)}

For equation (1.1) with several arguments, the following results have been established.

In 1982, Ladas and Stavroulakis [28] (see also in 1984, Arino et al. [5]) studied the equation with several constant retarded arguments of the form

$$
x^{\prime}(t)+\sum_{i=1}^{m} p_{i}(t) x\left(t-\tau_{i}\right)=0, \quad t \geq t_{0}
$$


under the assumption that $\liminf _{t \rightarrow \infty} \int_{t-\tau_{i} / 2}^{t} p(s) d s>0, i=1,2, \ldots, m$, and proved that each one of the following conditions

$$
\begin{aligned}
& \liminf _{t \rightarrow \infty} \int_{t-\tau_{i}}^{t} p_{i}(s) d s>\frac{1}{e} \text { for some } i, i=1,2, \ldots, m, \\
& \liminf _{t \rightarrow \infty} \int_{t-\tau}^{t} \sum_{i=1}^{m} p_{i}(s) d s>\frac{1}{e}, \quad \text { where } \tau=\min \left\{\tau_{1}, \tau_{2,}, \ldots, \tau_{m}\right\}, \\
& {\left[\prod_{i=1}^{m}\left(\sum_{j=1}^{m} \liminf _{t \rightarrow \infty} \int_{t-\tau_{j}}^{t} p_{i}(s) d s\right)\right]^{1 / m}>\frac{1}{e},}
\end{aligned}
$$

or

$$
\begin{aligned}
& \frac{1}{m} \sum_{i=1}^{m}\left(\liminf _{t \rightarrow \infty} \int_{t-\tau_{i}}^{t} p_{i}(s) d s\right) \\
& \quad+\frac{2}{m} \sum_{\substack{i<j \\
i, j=1}}^{m}\left[\left(\liminf _{t \rightarrow \infty} \int_{t-\tau_{j}}^{t} p_{i}(s) d s\right)\left(\liminf _{t \rightarrow \infty} \int_{t-\tau_{i}}^{t} p_{i}(s) d s\right)\right]^{\frac{1}{2}}>\frac{1}{e}
\end{aligned}
$$

implies that all solutions of equation (1.1') oscillate. Later in 1996, Li [19] proved that the same conclusion holds if

$$
\liminf _{t \rightarrow \infty} \sum_{i=1}^{m} \int_{t-\tau_{i}}^{t} p_{i}(s) d s>\frac{1}{e} .
$$

In 1984, Hunt and Yorke [29], considered the equation with variable coefficients of the form

$$
x^{\prime}(t)+\sum_{i=1}^{m} p_{i}(t) x\left(t-\tau_{i}(t)\right)=0, \quad t \geq t_{0}
$$

under the assumption that there is a uniform upper bound $\tau_{0}$ on the $\tau_{i}$ 's and proved that if

$$
\liminf _{t \rightarrow \infty} \sum_{i=1}^{m} \tau_{i}(t) p_{i}(t)>\frac{1}{e}
$$

then all solutions of equation (1.1") oscillate.

In 1984, Fukagai and Kusano [30], for equation (1.1), established the following theorem.

Theorem 3.1 ([30, Theorem $\left.\left.1^{\prime}(\mathrm{i})\right]\right)$ Consider equation (1.1) and assume that there is a continuous non-decreasing function $\tau^{*}(t)$ such that $\tau_{i}(t) \leq \tau^{*}(t) \leq t$ for $t \geq t_{0}, 1 \leq i \leq m$. If

$$
\liminf _{t \rightarrow \infty} \int_{\tau^{*}(t)}^{t} \sum_{i=1}^{m} p_{i}(s) d s>\frac{1}{e},
$$

then all solutions of equation (1.1) oscillate. If, on the other hand, there exists a continuous non-decreasing function $\tau_{*}(t)$ such that $\tau_{*}(t) \leq \tau_{i}(t)$ for $t \geq t_{0}, 1 \leq i \leq m, \lim _{t \rightarrow \infty} \tau_{*}(t)=\infty$ 
and

$$
\int_{\tau_{*}(t)}^{t} \sum_{i=1}^{m} p_{i}(s) d s \leq \frac{1}{e} \text { for all sufficiently large } t
$$

then equation (1.1) has a non-oscillatory solution.

In 2000, Grammatikopoulos et al. [31] improved the above results as follows.

Theorem 3.2 ([31, Theorem 2.6]) Assume that the functions $\tau_{i}$ are non-decreasing for all $i \in\{1, \ldots, m\}$,

$$
\int_{0}^{\infty}\left|p_{i}(t)-p_{j}(t)\right| d t<+\infty, \quad i, j=1, \ldots, m
$$

and

$$
\liminf _{t \rightarrow \infty} \int_{\tau_{i}(t)}^{t} p_{i}(s) d s=\beta_{i}>0, \quad i=1, \ldots, m
$$

If

$$
\sum_{i=1}^{m}\left(\liminf _{t \rightarrow \infty} \int_{\tau_{i}(t)}^{t} p_{i}(s) d s\right)>\frac{1}{e}
$$

then all solutions of equation (1.1) oscillate.

Observe that all the above mentioned oscillation conditions (3.1)-(3.7) involve liminf only and coincide with condition $\left(C_{2}\right)$ in the case of equation (1.2). It is obvious that there is a gap between conditions $\left(C_{1}\right)$ and $\left(C_{2}\right)$ when the $\operatorname{limit}_{t \rightarrow \infty} \int_{\tau(t)}^{t} p(s) d s$ does not exist. Moreover, in view of Theorem 2.3, it is an interesting problem to investigate equation (1.1) with non-monotone arguments and derive sufficient oscillation conditions, involving limsup (as condition $\left(C_{1}\right)$ for equation (1.2) with one argument), which is the main objective in the following.

Theorem 3.3 ([32]) Assume that there exist non-decreasing functions $\sigma_{i} \in C\left(\left[t_{0},+\infty\right)\right)$ such that

$$
\tau_{i}(t) \leq \sigma_{i}(t) \leq t \quad(i=1, \ldots, m)
$$

and

$$
\begin{array}{r}
\limsup _{t \rightarrow+\infty} \prod_{j=1}^{m}\left[\prod _ { i = 1 } ^ { m } \int _ { \sigma _ { j } ( t ) } ^ { t } p _ { i } ( s ) \operatorname { e x p } \left(\int_{\tau_{i}(s)}^{\sigma_{i}(t)} \sum_{i=1}^{m} p_{i}(\xi)\right.\right. \\
\left.\left.\quad \times \exp \left(\int_{\tau_{i}(\xi)}^{\xi} \sum_{i=1}^{m} p_{i}(u) d u\right) d \xi\right) d s\right]^{\frac{1}{m}}>\frac{1}{m^{m}} .
\end{array}
$$

Then all solutions of equation (1.1) oscillate. 
When $m=1$, that is, in the case of equation (1.2) with one argument, from Theorem 3.3 we have the following corollary.

Corollary 3.1 ([32]) Let

$$
C:=\limsup _{t \rightarrow+\infty} \int_{\sigma(t)}^{t} p(s) \exp \left(\int_{\tau(s)}^{\sigma(t)} p(\xi) \exp \left(\int_{\tau(\xi)}^{\xi} p(u) d u\right) d \xi\right) d s>1 .
$$

Then all solutions of equation (1.2) oscillate.

In the case of monotone arguments we have the following.

Theorem 3.4 ([32]) Let $\tau_{i}$ be non-decreasing functions and

$$
\begin{aligned}
& \limsup _{t \rightarrow+\infty} \prod_{j=1}^{m}\left[\prod _ { i = 1 } ^ { m } \int _ { \tau _ { j } ( t ) } ^ { t } p _ { i } ( s ) \operatorname { e x p } \left(\int_{\tau_{i}(s)}^{\tau_{i}(t)} \sum_{i=1}^{m} p_{i}(\xi)\right.\right. \\
& \left.\left.\quad \times \exp \left(\int_{\tau_{i}(\xi)}^{\xi} \sum_{i=1}^{m} p_{i}(u) d u\right) d \xi\right) d s\right]^{\frac{1}{m}}>\frac{1}{m^{m}} .
\end{aligned}
$$

Then all solutions of equation (1.1) oscillate.

Corollary 3.2 ([32]) Let $\tau_{i}$ be non-decreasing functions and

$$
\limsup _{t \rightarrow+\infty} \prod_{j=1}^{m}\left(\prod_{i=1}^{m} \int_{\tau_{j}(t)}^{t} p_{i}(s) d s\right)^{\frac{1}{m}}>\frac{1}{m^{m}}
$$

then all solutions of equation (1.1) oscillate.

Corollary 3.3 ([32]) Let $\tau_{i}$ be non-decreasing functions, $p_{i}(t) \geq p(t)(i=1, \ldots, m)$ and

$$
\limsup _{t \rightarrow+\infty} \prod_{j=1}^{m} \int_{\tau_{j}(t)}^{t} p(s) d s>\frac{1}{m^{m}}
$$

then all solutions of equation (1.1) oscillate.

Corollary 3.4 ([32]) Let $\tau_{i}$ be non-decreasing functions, $p_{i}(t) \geq p=$ const and

$$
p^{m} \limsup _{t \rightarrow+\infty} \prod_{i=1}^{m}\left(t-\tau_{i}(t)\right)>\frac{1}{m^{m}}
$$

then all solutions of equation (1.1) oscillate.

Remark 3.1 It should be pointed out that condition (3.9) of Theorem 3.3 presents for the first time sufficient conditions (in terms of lim sup) for the oscillation of all solutions to equation (1.1) with several non-monotone arguments. They are also independent and essentially improve all the related oscillation conditions in the literature. Even in the case where $m=1$, the improvement is substantial. Observe that (3.10) essentially improves (2.5). 
Remark 3.2 Observe that when $m=1$, the above condition (3.11) [(3.12)] reduces to the (classical) condition $\left(C_{1}\right)$.

The following examples illustrate the significance of our results.

Example 3.1 ( $c f .[7,32])$ We consider a generalization of an example presented in [7], where the equation

$$
x^{\prime}(t)+\frac{1}{e} x(\tau(t))=0, \quad t \geq 0,
$$

with the retarded argument

$$
\tau(t):= \begin{cases}t-1, & t \in[3 n, 3 n+1], \\ -3 t+(12 n+3), & t \in[3 n+1,3 n+2], \\ 5 t-(12 n+13), & t \in[3 n+2,3 n+3],\end{cases}
$$

was studied. Here we discuss the more general equation

$$
x^{\prime}(t)+p x(\tau(t))=0, \quad t \geq 0, p>0,
$$

and illustrate how our methodology can be utilized to prove the existence of oscillatory solutions for some range of the parameter $p$. In this case, as in [7], one may choose the function

$$
\sigma(t)= \begin{cases}t-1, & t \in[3 n, 3 n+1], \\ 3 n, & t \in[3 n+1,3 n+2.6], \\ 5 t-(12 n+13), & t \in[3 n+2.6,3 n+3] .\end{cases}
$$

Now note that, since $\tau(t) \leq t-1$,

$$
\int_{\tau(t)}^{t} p d u \geq \int_{t-1}^{t} p d u=p
$$

The choice as in [6] of $t_{n}=3 n+3$ gives

$$
\begin{aligned}
C & =\limsup _{t \rightarrow+\infty} \int_{\sigma(t)}^{t} p \exp \left(\int_{\tau(s)}^{\sigma(t)} p \exp \left(\int_{\tau(\xi)}^{\xi} p d u\right) d \xi\right) d s \\
& \geq \lim _{n \rightarrow+\infty} \int_{3 n+2}^{3 n+3} p \exp \left(\int_{5 s-(12 n+13)}^{3 n+2} p \exp (p) d \xi\right) d s=\frac{1}{5}\left(e^{5 p e^{p}}-1\right) e^{-p} .
\end{aligned}
$$

The inequality

$$
\frac{1}{5}\left(e^{5 p e^{p}}-1\right) e^{-p}>1
$$

is satisfied for (the numbers that follow are rounded to the third decimal place unless exact) $p \in[0.303,0.358]$. Thus, for $p \in[0.303,0.358]$, condition (3.10) of Corollary 3.1 is satisfied, 
and therefore all solutions to the above equation (3.14) oscillate. Observe, however, that when $p \in[0.303,0.358]$ in (3.14), we find

$$
\begin{aligned}
& A=\limsup _{t \rightarrow \infty} \int_{\sigma(t)}^{t} p d s=p \cdot(2.6)<1, \\
& \mathfrak{a}:=\liminf _{t \rightarrow \infty} \int_{\tau(t)}^{t} p d s=p<\frac{1}{e}
\end{aligned}
$$

and

$$
\begin{aligned}
\int_{\sigma(3 n+3)}^{3 n+3} p \exp \left\{\int_{\tau(s)}^{\sigma(3 n+3)} p d \xi\right\} d s & =\int_{3 n+2}^{3 n+3} p \exp \left\{\int_{5 s-(12 n+13)}^{3 n+2} p d \xi\right\} d s \\
& =\frac{1}{5}\left(e^{5 p}-1\right)<1 .
\end{aligned}
$$

That is, none of the known oscillation conditions $\left(C_{1}\right),\left(C_{2}\right)$ (and also conditions (2.5), (3.1)-(3.6)) is satisfied.

Remark 3.3 ([32]) It is obvious that if for some $i_{0} \in\{1, \ldots, m\}$ all solutions of the equation

$$
x^{\prime}(t)+p_{i_{0}}(t) x\left(\tau_{i_{0}}(t)\right)=0
$$

oscillate, then all solutions of equation (1.1) also oscillate.

Example 3.2 ([32]) Let $p, \Delta_{1}, \Delta_{2} \in(0,+\infty)$ and consider the sequences $\left\{t_{k}\right\}_{k=1}^{\infty}$ such that $t_{k} \uparrow+\infty$ for $k \uparrow+\infty, t_{k}+2 \Delta<t_{k+1}(k=1,2, \ldots)$, where $\Delta=\max \left\{\Delta_{i}, i=1,2\right\}$. Choose $p, \Delta_{1}$ and $\Delta_{2}$ such that

$$
p^{2} \Delta_{1} \Delta_{2}>\frac{1}{4}
$$

and

$$
p \Delta_{i}<1 \quad(i=1,2) .
$$

Let $p(t)=p$ for $t \in\left[t_{k}, t_{k}+\Delta\right](k=1,2, \ldots)$ and $p(t)=0$ for $t \in R_{+} \backslash \bigcup_{k=1}^{\infty}\left[t_{k}, t_{k}+\Delta\right]$.

According to (3.15), it is obvious that condition (3.13) is fulfilled, where $m=2$ and $\tau_{i}(t)=$ $t-\Delta_{i}(i=1,2)$ a.e., and therefore all solutions to equation (1.1) are oscillatory. However, for the equations

$$
x^{\prime}(t)+p(t) x\left(t-\Delta_{i}\right)=0 \quad(i=1,2)
$$

by (3.16), we have

$$
\limsup _{t \rightarrow+\infty} \int_{t-\Delta_{i}}^{t} p(s) d s<1 \quad(i=1,2)
$$

and

$$
\liminf _{t \rightarrow+\infty} \int_{t-\Delta_{i}}^{t} p(s) d s=0 \quad(i=1,2) .
$$


Remark 3.4 ([32]) In the above mentioned Example 3.2, by a solution we mean an absolutely continuous function which satisfies the corresponding equation almost everywhere.

Example 3.3 ([32]) Consider the equation

$$
x^{\prime}(t)+p_{1} x\left(\tau_{1}(t)\right)+p_{2} x\left(\tau_{2}(t)\right)=0, \quad t \geq 0, p_{1}, p_{2}>0,
$$

where

$$
\begin{gathered}
\tau_{1}(t)= \begin{cases}t-1, & t \in[3 n, 3 n+1], \\
-3 t+(12 n+3), & t \in[3 n+1,3 n+2], \\
5 t-(12 n+13), & t \in[3 n+2,3 n+3],\end{cases} \\
\tau_{2}(t)= \begin{cases}t-2, & t \in[3 n, 3 n+1], \\
-t+6 n, & t \in[3 n+1,3 n+2], \\
3 t-(6 n+8), & t \in[3 n+2,3 n+3] .\end{cases}
\end{gathered}
$$

We can take

$$
\begin{aligned}
& \sigma_{1}(t)= \begin{cases}t-1, & t \in[3 n, 3 n+1], \\
3 n, & t \in[3 n+1,3 n+2.6], \\
5 t-(12 n+13), & t \in[3 n+2.6,3 n+3],\end{cases} \\
& \sigma_{2}(t)= \begin{cases}t-2, & t \in[3 n, 3 n+1], \\
3 n-1, & t \in[3 n+1,3 n+2 . \overline{3}], \\
3 t-(6 n+8), & t \in[3 n+2 . \overline{3}, 3 n+3] .\end{cases}
\end{aligned}
$$

Note that, since $\tau_{1}(t) \leq t-1$ and $\tau_{2}(t) \leq t-2$, we have

$$
\int_{\tau_{1}(t)}^{t} d u \geq \int_{t-1}^{t} d u=1, \quad \int_{\tau_{2}(t)}^{t} d u \geq \int_{t-2}^{t} d u=2 .
$$

Set $P=p_{1} \exp \left(p_{1}+p_{2}\right)+p_{2} \exp \left(2 p_{1}+2 p_{2}\right)$. The choice of $t_{n}=3 n+3$ gives

$$
\begin{aligned}
\limsup _{t \rightarrow+\infty} & \prod_{j=1}^{2}\left(\prod_{i=1}^{2} \int_{\sigma_{j}(t)}^{t} p_{i} \exp \left(\int_{\tau_{i}(s)}^{\sigma_{i}(t)} \sum_{i=1}^{2} p_{i} \exp \left(\int_{\tau_{i}(\xi)}^{\xi}\left(p_{1}+p_{2}\right) d u\right) d \xi\right) d s\right)^{\frac{1}{2}} \\
\geq & \lim _{n \rightarrow+\infty} \prod_{j=1}^{2}\left(\prod_{i=1}^{2} \int_{\sigma_{j}(3 n+3)}^{3 n+3} p_{i} \exp \left(\int_{\tau_{i}(s)}^{\sigma_{i}(3 n+3)} \sum_{i=1}^{2} p_{i} \exp \left(\int_{\tau_{i}(\xi)}^{\xi}\left(p_{1}+p_{2}\right) d u\right) d \xi\right) d s\right)^{\frac{1}{2}} \\
\geq & \lim _{n \rightarrow+\infty} \prod_{j=1}^{2}\left(\int_{\sigma_{j}(3 n+3)}^{3 n+3} p_{1} \exp \left(\int_{\tau_{1}(s)}^{3 n+2} P d \xi\right) d s\right)^{\frac{1}{2}} \\
& \times\left(\int_{\sigma_{j}(3 n+3)}^{3 n+3} p_{2} \exp \left(\int_{\tau_{2}(s)}^{3 n+1} P d \xi\right) d s\right)^{\frac{1}{2}} \\
= & \lim _{n \rightarrow+\infty}\left(\int_{3 n+2}^{3 n+3} p_{1} \exp \left(\int_{\tau_{1}(s)}^{3 n+2} P d \xi\right) d s\right)^{\frac{1}{2}} \times\left(\int_{3 n+2}^{3 n+3} p_{2} \exp \left(\int_{\tau_{2}(s)}^{3 n+1} P d \xi\right) d s\right)^{\frac{1}{2}}
\end{aligned}
$$




$$
\begin{aligned}
& \times\left(\int_{3 n+1}^{3 n+3} p_{1} \exp \left(\int_{\tau_{1}(s)}^{3 n+2} P d \xi\right) d s\right)^{\frac{1}{2}} \times\left(\int_{3 n+1}^{3 n+3} p_{2} \exp \left(\int_{\tau_{2}(s)}^{3 n+1} P d \xi\right) d s\right)^{\frac{1}{2}} \\
= & \lim _{n \rightarrow+\infty}\left(\int_{3 n+2}^{3 n+3} p_{1} \exp \left(\int_{5 s-(12 n+13)}^{3 n+2} P d \xi\right) d s\right)^{\frac{1}{2}} \\
& \times\left(\int_{3 n+2}^{3 n+3} p_{2} \exp \left(\int_{3 s-(6 n+8)}^{3 n+1} P d \xi\right) d s\right)^{\frac{1}{2}} \\
& \times\left(\int_{3 n+1}^{3 n+2} p_{1} \exp \left(\int_{-3 s+(12 n+3)}^{3 n+2} P d \xi\right) d s+\int_{3 n+2}^{3 n+3} p_{1} \exp \left(\int_{5 s-(12 n+13)}^{3 n+2} P d \xi\right) d s\right)^{\frac{1}{2}} \\
& \times\left(\int_{3 n+1}^{3 n+2} p_{2} \exp \left(\int_{-s+6 n}^{3 n+1} P d \xi\right) d s+\int_{3 n+2}^{3 n+3} p_{2} \exp \left(\int_{3 s-(6 n+8)}^{3 n+1} P d \xi\right) d s\right)^{\frac{1}{2}} \\
= & D\left(p_{1}, p_{2}\right) .
\end{aligned}
$$

Let $p_{1}=0.1$. Then, by direct computation, we get

$$
D>\frac{1}{4}
$$

if $p_{2} \geq 0.158$. That is, when $p_{1}=0.1$ and $p_{2} \geq 0.158$ in equation (3.17), condition (3.9) of Theorem 3.3 is satisfied, and therefore all solutions to this equation oscillate.

Note that since the delays are not monotone, Theorem 3.2 cannot be applied to this example. We now compare our result with Theorem 3.1. Note that

$$
\tau_{1}(t), \tau_{2}(t) \leq \sigma_{1}(t) \quad \text { for every } t>0
$$

The choice $p_{1}=0.1, p_{2}=0.158$ gives

$$
\liminf _{t \rightarrow \infty} \int_{\sigma_{1}(t)}^{t}\left(p_{1}+p_{2}\right) d s=p_{1}+p_{2}=0.258<\frac{1}{e}
$$

that is, condition (3.6) is not satisfied.

\section{Competing interests}

The author declares that he has no competing interests.

Received: 11 July 2014 Accepted: 26 September 2014 Published: 16 Oct 2014

\section{References}

1. Erbe, LH, Kong, Q, Zhang, BG: Oscillation Theory for Functional Differential Equations. Dekker, New York (1995)

2. Gopalsamy, K: Stability and Oscillations in Delay Differential Equations of Population Dynamics. Kluwer Academic, Dordrecht (1992)

3. Gyori, I, Ladas, G: Oscillation Theory of Delay Differential Equations with Applications. Clarendon, Oxford (1991)

4. Hale, JK: Theory of Functional Differential Equations. Springer, New York (1997)

5. Arino, O, Gyori, I, Jawhari, A: Oscillation criteria in delay equations. J. Differ. Equ. 53, 115-123 (1984)

6. Berezansky, L, Braverman, E: On some constants for oscillation and stability of delay equations. Proc. Am. Math. Soc. 139(11), 4017-4026 (2011)

7. Braverman, E, Karpuz, B: On oscillation of differential and difference equations with non-monotone delays. Appl. Math. Comput. 58, 766-775 (2011)

8. Elbert, A, Stavroulakis, IP: Oscillations of first order differential equations with deviating arguments. In: Recent Trends in Differential Equations. Univ. of loannina T.R. No. 172 (1990). World Sci. Ser. Appl. Anal., vol. 1, pp. 163-178. World Sci. Publ., River Edge (1992)

9. Elbert, A, Stavroulakis, IP: Oscillation and non-oscillation criteria for delay differential equations. Proc. Am. Math. Soc 123, 1503-1510 (1995) 
10. Erbe, LH, Zhang, BG: Oscillation of first order linear differential equations with deviating arguments. Differ. Integral Equ. 1, 305-314 (1988)

11. Jaroš, J, Stavroulakis, IP: Oscillation tests for delay equations. Rocky Mt. J. Math. 29, 139-145 (1999)

12. Jian, C: Oscillation of linear differential equations with deviating argument. Math. Pract. Theory 1, 32-41 (1991) (in Chinese)

13. Kon, M, Sficas, YG, Stavroulakis, IP: Oscillation criteria for delay equations. Proc. Am. Math. Soc. 128, $2989-2997$ (2000)

14. Koplatadze, RG, Chanturija, TA: On the oscillatory and monotonic solutions of first order differential equations with deviating arguments. Differ. Uravn. 18, 1463-1465 (1982)

15. Koplatadze, RG, Kvinikadze, G: On the oscillation of solutions of first order delay differential inequalities and equations. Georgian Math. J. 1, 675-685 (1994)

16. Kwong, MK: Oscillation of first order delay equations. J. Math. Anal. Appl. 156, 286-374 (1991)

17. Ladas, G: Sharp conditions for oscillations caused by delay. Appl. Anal. 9, 93-98 (1979)

18. Ladde, GS, Lakshmikantham, V, Zhang, BG: Oscillation Theory of Differential Equations with Deviating Arguments. Dekker, New York (1987)

19. Li, B: Oscillations of first order delay differential equations. Proc. Am. Math. Soc. 124, 3729-3737 (1996)

20. Myshkis, AD: Linear homogeneous differential equations of first order with deviating arguments. Usp. Mat. Nauk 5 , 160-162 (1950) (in Russian)

21. Philos, CG, Sficas, YG: An oscillation criterion for first-order linear delay differential equations. Can. Math. Bull. 41, 207-213 (1998)

22. Sficas, YG, Stavroulakis, IP: Oscillation criteria for first-order delay equations. Bull. Lond. Math. Soc. 35, $239-246$ (2003)

23. Stavroulakis, IP: Oscillation criteria for delay and difference equations with non-monotone arguments. Appl. Math. Comput. 226, 661-672 (2014)

24. Wang, ZC, Stavroulakis, IP, Qian, XZ: A survey on the oscillation of solutions of first order linear differential equations with deviating arguments. Appl. Math. E-Notes 2, 171-191 (2002)

25. Yu, JS, Wang, ZC, Zhang, BG, Qian, XZ: Oscillations of differential equations with deviating arguments. Panam. Math. J. 2, 59-78 (1992)

26. Zhou, Y, Yu, YH: On the oscillation of solutions of first order differential equations with deviating arguments. Acta Math. Appl. Sin. 15(3), 288-302 (1999)

27. Ladas, G, Laskhmikantham, V, Papadakis, JS: Oscillations of higher-order retarded differential equations generated by retarded arguments. In: Delay and Functional Differential Equations and Their Applications, pp. 219-231. Academic Press, New York (1972)

28. Ladas, G, Stavroulakis, IP: Oscillations caused by several retarded and advanced arguments. J. Differ. Equ. 44, 134-152 (1982)

29. Hunt, BR, Yorke, JA: When all solutions of $x^{\prime}=\sum q_{i}(t) x\left(t-T_{i}(t)\right)$ oscillate. J. Differ. Equ. 53, 139-145 (1984)

30. Fukagai, N, Kusano, T: Oscillation theory of first order functional differential equations with deviating arguments. Ann. Mat. Pura Appl. 136, 95-117 (1984)

31. Grammatikopoulos, MK, Koplatadze, RG, Stavroulakis, IP: On the oscillation of solutions of first order differential equations with retarded arguments. Georgian Math. J. 10,63-76 (2003)

32. Infante, G, Koplatadze, R, Stavroulakis, IP: Oscillation criteria for differential equations with several retarded arguments. Funkc. Ekvacioj (to appear). arXiv:1309.6213

10.1186/1029-242X-2014-399

Cite this article as: Stavroulakis: A survey on the oscillation of differential equations with several deviating arguments. Journal of Inequalities and Applications 2014, 2014:399

\section{Submit your manuscript to a SpringerOpen ${ }^{\ominus}$ journal and benefit from:}

- Convenient online submission

- Rigorous peer review

- Immediate publication on acceptance

- Open access: articles freely available online

- High visibility within the field

- Retaining the copyright to your article 